\title{
Practical consensus of homogeneous sampled-data multi-agent systems
}

\author{
Emmanuel Bernuau, Emmanuel Moulay, Patrick Coirault, and Fayrouz Isfoula
}

\begin{abstract}
The aim of this article is to study the secondorder practical consensus of homogeneous sampled-data multiagent systems. To do this, a new nonlinear emulation strategy based on homogeneity is developed. It is then applied to multiagent systems under synchronously variable sampling. Finally, a comparison with the classical linear strategy is provided in the case of multi-agent systems under synchronously periodic sampling.
\end{abstract}

Index Terms-Multi-agent systems, practical consensus, sampled-data systems, homogeneous systems.

\section{INTRODUCTION}

The second-order consensus for multi-agent systems (MAS) has been solved during the 2000's by using linear controllers [1]. The multi-agent formation control has then attracted a lot of attention [2]. For real applications involving wireless communications, the problem of the sampled-data inputs must be taken into account [3]. So, the study of sampled-data MAS has been developed in the 2010's [4], [5], [6], [7], [8], [9], [10], [11], [12]. There are four kinds of samplings that appears for MAS: synchronously periodic sampling (SPS) where all agents have the same periodic sampling on the input; asynchronously periodic sampling (APS) where each agent has its own periodic sampling on the input; synchronously variable sampling (SVS) where all agents have the same aperiodic sampling on the input; and asynchronously variable sampling (AVS) where each agent has its own aperiodic sampling on the input. The reader can find an overview on sampled-data MAS in [13], [14].

To tackle the problem of stability of the double integrator with a sampled-data input, a new nonlinear emulation strategy based on homogeneous systems has been proposed in [15]. With the emulation method, the controller is first designed in continuous time and then implemented as a sampled-data controller [16], [3], [17]. The strategy proposed in [15] for homogeneous nonlinear systems with sampled-data inputs cannot be directly used for MAS. Indeed, this paper only considers control laws leading to asymptotic stability in continuous time, whereas the consensus of MAS do not lead to asymptotic stability in continuous time.

E. Bernuau is with UMR 1145 Ingénierie Procédés Aliments, AgroParisTech, 1 Avenue des Olympiades, 91744 Massy Cedex, France (e-mail: emmanuel.bernuau@agroparistech.fr).

E. Moulay is with XLIM (UMR CNRS 7252), Université de Poitiers, 11 bd Marie et Pierre Curie, 86962 Futuroscope Chasseneuil Cedex, France (e-mail: emmanuel.moulay@univ-poitiers.fr).

P. Coirault and F. Isfoula are with LIAS (EA 6315), Université de Poitiers, 2 rue Pierre Brousse, 86073 Poitiers Cedex 9, France (e-mail: fayrouz.isfoula@univ-poitiers.fr, patrick.coirault@univ-poitiers.fr).

Manuscript received April 19, 2005; revised August 26, 2015.
The first task of this article is to adapt the result of [15] and prove a new theoretical result for the set stability of homogeneous nonlinear systems with sampled-data inputs and relevant for MAS. Then, we use this new result to obtain the practical consensus for homogeneous sampled-data MAS [18] with undirected or directed graphs. We only ensure practical consensus due to the sampling of the inputs which can be arbitrary large. In practice, we need a controller for the continuous plant which renders the closed-loop MAS homogeneous of negative degree. This is precisely what is done by some finite time controllers by using the notion of finite time stability developed in [19]. The finite time consensus of MAS has been studied for instance in [20], [21] for double-integrator dynamics. In our article, we use the homogeneous nonlinear controllers proposed in [21], [22] in order to obtain the practical consensus of the second-order MAS with SVS, which has been studied with linear controllers in [23]. The main advantage of the proposed method relies on the fact that the maximum distance between the agents remains bounded when the sampling interval increases, unlike linear strategies. The main drawback is the loss of the exact consensus, replaced by a practical consensus; however, the maximal distance between agents is bounded by an explicit function on the maximum sampling period. The results are illustrated in simulations with the controller proposed in [22] in order to compare with emulated linear strategies, which fail to maintain the consensus when the sampling periods are too high.

The article is organized as follows. After some notations, definitions and preliminary results given in Section II, the main results are addressed in Section III. Then, they are illustrated in simulation in Section IV. Finally, a conclusion is provided in Section V.

\section{PRELIMINARY}

Let us introduce the following notations:

- $\mathbb{R}_{+}=\{x \in \mathbb{R}: x \geq 0\}$, where $\mathbb{R}$ is the set of real numbers.

- $|\cdot|$ denotes the absolute value in $\mathbb{R}$, for $p \geq 1,\|\cdot\|_{p}$ denotes the $p$-norm on $\mathbb{R}^{n}$ and $\|\cdot\|=\|\cdot\|_{2}$ denotes the Euclidean norm on $\mathbb{R}^{n}$.

- For any closed set $Z \subset \mathbb{R}^{n}$ and any $x \in \mathbb{R}^{n}$, we denote $\mathrm{d}_{Z}(x)=\inf _{z \in Z}\|x-z\|$ the distance between $x$ and the closed set $Z$.

- If $x(t)$ is a curve in $\mathbb{R}^{n}$, we will say that $x(t) \rightarrow Z$ as $t \rightarrow+\infty$ when $\mathrm{d}_{Z}(x(t)) \rightarrow 0$ as $t \rightarrow+\infty$.

- We denote $1_{n}=(1, \ldots, 1)^{T} \in \mathbb{R}^{n}$ and $\Delta_{n}=$ $\operatorname{Span}\left(1_{n}\right) \subset \mathbb{R}^{n}$. 
- $\mathbf{r}=\left(r_{1}, \ldots, r_{n}\right)$ is called a generalized weight if its components are positive numbers.

- For any generalized weight $\mathbf{r}$ and for any $\lambda>$ 0 , we denote $\Lambda_{r}(\lambda)=\operatorname{Diag}\left(\lambda^{r_{1}}, \ldots, \lambda^{r_{n}}\right)$ where $\operatorname{Diag}\left(a_{1}, \ldots, a_{n}\right)$ denotes the diagonal matrix of dimension $n \times n$ with $k^{t h}$ diagonal entry $a_{k}$.

- $\lfloor x\rceil^{\alpha}=\left(\left|x_{1}\right|^{\alpha} \operatorname{sign}\left(x_{1}\right), \ldots,\left|x_{n}\right|^{\alpha} \operatorname{sign}\left(x_{n}\right)\right)^{T}$ where $x=\left(x_{1}, \ldots, x_{n}\right)^{T} \in \mathbb{R}^{n}$ and $\alpha>0$.

- If $A$ is an $m \times n$ matrix and $B$ a $p \times q$ matrix, then the Kronecker product $A \otimes B$ is the $m p \times n q$ block matrix

$$
A \otimes B=\left(\begin{array}{ccc}
a_{11} B & \cdots & a_{1 n} B \\
\vdots & \ddots & \vdots \\
a_{m 1} B & \cdots & a_{m n} B
\end{array}\right) .
$$

- $I_{n}$ denotes the unit matrix of size $n$.

- If $V \subset \mathbb{R}^{n}$, we define the orthogonal complement of $V$ by $V^{\perp}=\left\{x \in \mathbb{R}^{n}: x^{T} y=0\right.$ for all $\left.y \in V\right\}$.

Definition 1: Let $\eta$ and $h$ be positive numbers. An $(\eta, h)$ sampling sequence is a sequence $\left(t_{k}\right)_{k \geq 0}$ such that $t_{0}=0$ and for all $k \geq 0, \eta \leq t_{k+1}-t_{k} \leq h$.

\section{A. Graph theory}

Let us recall some basic definitions about graph theory given for instance in [1, Appendix B].

A directed graph $\mathcal{G}_{N}=\left(V_{N}, \mathcal{E}_{N}\right)$ consists of a finite nonempty set of nodes $V_{N}=\{1,2, \ldots, N\}$ and a set of edges $\mathcal{E}_{N} \subset V_{N} \times V_{N}$ which is a set of ordered pairs of nodes. An edge $(i, j) \in \mathcal{E}_{N}$ in a directed graph $\mathcal{G}_{N}$ denotes that node $i$ communicates with node $j$, but not conversely. An undirected graph $\mathcal{G}_{N}=\left(V_{N}, \mathcal{E}_{N}\right)$ also consists of a set of nodes $V_{N}=\{1, \ldots, N\}$ and a set of edges $\mathcal{E}_{N} \subset V_{N} \times V_{N}$ which is an unordered set of pairs of nodes. An edge $(i, j) \in$ $\mathcal{E}_{N}$ in an undirected graph $\mathcal{G}_{N}$ denotes that nodes $i$ and $j$ obtain information from each other. An undirected path is a sequence of edges in an undirected graph of the form $\left(i_{1}, i_{2}\right)$, $\left(i_{2}, i_{3}\right), \cdots$. An undirected graph is connected if there is an undirected path between every pair of distinct nodes.

The adjacency matrix of an undirected graph $\left(V_{N}, \mathcal{E}_{N}\right)$ is defined by $\mathcal{A}_{N}=\left[a_{i j}\right] \in \mathbb{R}^{N \times N}$ where $a_{i j}=a_{j i}=1$ if $(i, j) \in \mathcal{E}_{N}$ and $a_{i j}=0$ otherwise. The Laplacian matrix associated with adjacency matrix $\mathcal{A}_{N}$ is given as $\mathcal{L}_{N}=\left[\ell_{i j}\right] \in$ $\mathbb{R}^{N \times N}$ where $\ell_{i i}=\sum_{j=1, j \neq i}^{N} a_{i j}$ and $\ell_{i j}=-a_{i j}$ if $i \neq j$.

\section{B. Lyapunov stability}

Consider the following system with $f$ continuous

$$
\dot{x}=f(x), \quad x \in \mathbb{R}^{n} .
$$

Let us extend to closed sets the definitions of Lyapunov set stability given for instance in [24] for compact sets.

Definition 2: A closed set $C \subset \mathbb{R}^{n}$ is:

- stable w.r.t. the system (1) if for any $\varepsilon>0$ there exists $\eta>0$ such that for any maximal solution $x(t)$ of (1), if there exists $t_{0}$ such that $\mathrm{d}_{C}\left(x\left(t_{0}\right)\right)<\eta$, then $x(t)$ is defined for all $t \geq t_{0}$ and $\mathrm{d}_{C}(x(t))<\varepsilon$ for all $t \geq t_{0}$;
- locally attractive w.r.t. the system (1) if there exists $\varepsilon>0$ such that for any maximal solution $x(t)$ of (1), if there exists $t_{0}$ such that $\mathrm{d}_{C}\left(x\left(t_{0}\right)\right)<\varepsilon$, then $x(t)$ is defined for all $t \geq t_{0}$ and $\mathrm{d}_{C}(x(t)) \rightarrow 0$ when $t \rightarrow+\infty$;

- globally attractive w.r.t. the system (1) if it is locally attractive and if the previous point holds for any $\varepsilon>0$;

- locally (resp. globally) asymptotically stable w.r.t. the system (1) if it is stable and locally (resp. globally) attractive w.r.t. the system (1);

- unstable if it is not stable.

\section{Homogeneity}

The most common notion of homogeneity is the weighted homogeneity introduced in [25], based on a particular choice of the coordinates, while the most generic one is the geometric homogeneity, which is coordinate free [26], [27]. We use in the sequel the framework of weighted homogeneity.

Definition 3: Let $\mathcal{V}$ be a finite dimensional vector space and $A: \mathcal{V} \rightarrow \mathcal{V}$ an endomorphism of $\mathcal{V}$ such that the real part of each eigenvalue of $A$ is positive. We will say that a function $V: \mathcal{V} \rightarrow \mathcal{V}$ is A-homogeneous of degree $d$ if $V(\exp (A s) x)=e^{d s} V(x)$, for all $s \in \mathbb{R}$ and $x \in \mathcal{V}$. A vector field $f$ defined on $\mathcal{V}$ is A-homogeneous of degree $d$ if $f(\exp (A s) x)=e^{d s} \exp (A s) f(x)$, for all $s \in \mathbb{R}$ and $x \in \mathbb{R}^{n}$. If $\mathbf{r}=\left(r_{1}, \ldots, r_{n}\right)$ is a generalized weight, we will simply say that a function or a vector field defined on $\mathbb{R}^{n}$ is r-homogeneous of degree $d$ if it is $A$-homogeneous of degree $d$ for the endomorphism defined for all $i=1, \ldots, n$ by $A\left(e_{i}\right)=r_{i} e_{i}$, where $e_{i}$ denotes the $i^{t h}$ vector of the canonical basis of $\mathbb{R}^{n}$.

Let us now give the definition of a homogeneous norm detailed for instance in [15].

Definition 4: A r-homogeneous norm is a positive definite and continuous mapping $\mathcal{N}: \mathbb{R}^{n} \rightarrow \mathbb{R}$ that is $\mathbf{r}$-homogeneous of degree 1 .

\section{Technical lemmas}

Lemma 5: Let $M$ be a square matrix of size $n$ and $K$ a compact subset of $\mathbb{R}^{n}$ such that $K \subset \operatorname{im} M$. Then for any curve $x(t) \in \mathbb{R}^{n}$, we have $M x(t) \rightarrow K$ if and only if $x(t) \rightarrow$ $M^{-1} K$. In particular, we have $M x(t) \rightarrow 0$ if and only if $x(t) \rightarrow \operatorname{ker} M$.

Proof. If $M x(t) \rightarrow K$, there exists $y(t) \in K$ such that $\mathrm{d}_{K}(M x)=\|M x-y\| \rightarrow 0$. Then, there exists $z(t) \in M^{-1} K$ such that $M z=y$ and $x-z \in(\operatorname{ker} M)^{\perp}$. Therefore $\| M(x-$ $z) \| \rightarrow 0$. Given that $M$ is an isomorphism when restricted to $(\operatorname{ker} M)^{\perp}$, we have $\|x-z\| \rightarrow 0$ and then $\mathrm{d}_{M^{-1} K}(x) \rightarrow 0$, that is $x(t) \rightarrow M^{-1} K$. Conversely, if $x(t) \rightarrow M^{-1} K$, there exists $z(t) \in M^{-1} K$ such that $\|x-z\| \rightarrow 0$. It yields that $\|M x-M z\| \rightarrow 0$, which, given that $M z \in K$, shows that $\mathrm{d}_{K}(M x) \rightarrow 0$, that is $M x(t) \rightarrow K$. The last statement is immediate taking $K=\{0\}$.

Lemma 6: For any curve $x(t) \in \mathbb{R}^{n}$ of coordinates $x_{i}(t)$, $i=1, \ldots, n$, we have $x(t) \rightarrow \Delta_{n}$ if and only if for all $i$ and $j$ we have $x_{i}(t)-x_{j}(t) \rightarrow 0$. 
Proof. Let us denote $\Pi$ the orthogonal projection matrix onto $\Delta_{n}^{\perp}$. We easily see that $\Pi=V V^{T}$ with $V \in \mathbb{R}^{n \times(n-1)}$ a matrix of columns denoted $v_{i}$ and given by

$$
v_{i}=\frac{1}{\sqrt{i+i^{2}}}\left(\begin{array}{c}
1 \\
\vdots \\
1 \\
-i \\
0 \\
\vdots \\
0
\end{array}\right)
$$

where the $-i$ is located on the $(i+1)^{t h}$ line. Lemma 5 yields $x(t) \rightarrow \Delta_{n}$ if and only if $\Pi x(t) \rightarrow 0$. But we also have $\Pi x \rightarrow 0$ iff $\forall i, v_{i}^{T} x \rightarrow 0$ iff $\forall i, j, x_{i}-x_{j} \rightarrow 0$, which concludes the proof.

\section{MAIN RESULTS}

Let us state the main theoretical result of this article.

Theorem 7: Let $\dot{x}=f(x, u)$ be a continuous controlled vector field. Assume that we know a continuous control $u(x)$ such that:

1) there exists a linear subspace $E$ such that for any $x_{1}, x_{2}, z_{1}, z_{2} \in \mathbb{R}^{n}$, if $x_{1}-x_{2} \in E$ and $z_{1}-z_{2} \in E$, then $f\left(x_{1}, u\left(z_{1}\right)\right)-f\left(x_{2}, u\left(z_{2}\right)\right) \in E$;

2 ) the set $E$ is globally asymptotically stable for $\dot{x}=$ $f(x, u(x))$;

3 ) there exists an endomorphism of $\mathbb{R}^{n}$ denoted $A$ such that:

- the real part of each eigenvalue of $A$ is positive;

- the linear subspaces $E$ and $E^{\perp}$ are invariant subspaces of $A$;

- there exists $\kappa<0$ such that for all $x, z \in \mathbb{R}^{n}$ and all $s \in \mathbb{R}$ we have $f(\exp (A s) x, u(\exp (A s) z))=$ $e^{\kappa s} \exp (A s) f(x, u(z))$.

Then, for any $(\eta, h)$-sampling sequence $\left(t_{k}\right)_{k \in \mathbb{N}}$ and any $\tilde{A}$ homogeneous norm $\mathcal{N}$, there exists $C>0$ such that any solution $x(t)$ of

$$
\dot{x}(t)=f\left(x(t), u\left(x\left(t_{k}\right)\right)\right), \quad t \in\left[t_{k}, t_{k+1}\right)
$$

converges to $\left\{\xi \in E^{\perp}: \mathcal{N}(\xi) \leq C h^{-1 / \kappa}\right\}+E$, where $\tilde{A}$ denotes the restriction of $A$ to $E^{\perp}$. From assumption 3, we have $A \circ \pi=\pi \circ A$.

We define, for $\xi_{1}, \xi_{2} \in E^{\perp}$ the controlled vector field $g\left(\xi_{1}, u\left(\xi_{2}\right)\right)=\pi\left(f\left(\xi_{1}, u\left(\xi_{2}\right)\right)\right)$. Remark that assumption 1 yields that, for any $\zeta_{1}, \zeta_{2} \in E$, we have $g\left(\xi_{1}, u\left(\xi_{2}\right)\right)=$ $g\left(\xi_{1}+\zeta_{1}, u\left(\xi_{2}+\zeta_{2}\right)\right)$. Let us first show that we can apply Theorem 12 in [15] to $g$.

From assumption 3, for $\xi_{1}$ and $\xi_{2} \in E^{\perp}$ we get
Proof. We will denote $\pi$ the orthogonal projection onto $E^{\perp}$.

Consider $\xi(t) \in E^{\perp}$ a solution of $\dot{\xi}=g(\xi, u(\xi))$ such that $\xi(0)=\xi_{0} \in E^{\perp}$. The vector field being continuous, $\xi$ is absolutely continuous ${ }^{1}$. Now, denoting $p_{E}^{\perp}$ the orthogonal projection onto $E$, consider a solution of $\dot{\zeta}=p_{E}^{\perp} f(\xi+\zeta, u(\xi+$ $\zeta))$ such that $\zeta(0)=0$. Clearly, $\zeta(t) \in E$.

The curve $\xi(t)+\zeta(t)$ is a solution of $\dot{x}=f(x, u(x))$. Indeed, $\dot{\xi}+\dot{\zeta}=g(\xi, u(\xi))+p_{E}^{\perp} f(\xi+\zeta, u(\xi+\zeta))=$ $\pi f(\xi+\zeta, u(\xi+\zeta))+p_{E}^{\perp} f(\xi+\zeta, u(\xi+\zeta))=f(\xi+\zeta, u(\xi+\zeta))$. This shows that, for any solution $\xi(t) \in E^{\perp}$ of $\dot{\xi}=g(\xi, u(\xi))$, there exists a solution $x$ of $\dot{x}=f(x, u(x))$ such that $\pi x=\xi$.

Now, considering a curve $x(t) \in \mathbb{R}^{n}$ such that $\pi x(t)=\xi(t)$ and $\dot{x}=f(x, u(x))$, by assumption 2 we have $x(t) \rightarrow E$ and Lemma 5 yields that $\xi(t)=\pi x(t) \rightarrow 0$. We conclude that the vector field defined by $\dot{\xi}=g(\xi, u(\xi))$ is $\tilde{A}$-homogeneous and $0 \in E^{\perp}$ is a globally attractive equilibrium, which implies it is a globally asymptotically stable equilibrium. Thus, we can apply Theorem 12 in [15] and there exists a constant $C>0$ such that the solution of

$$
\dot{\xi}(t)=g\left(\xi(t), u\left(\xi\left(t_{k}\right)\right)\right), \quad t \in\left[t_{k}, t_{k+1}\right)
$$

converges to the compact set $\left\{\xi \in E^{\perp}: \mathcal{N}(\xi) \leq C h^{-1 / \kappa}\right\}$. Finally, given a solution $x(t)$ of (2), by assumption $1, \pi x(t)$ is a solution of (3) and therefore $\pi x(t) \rightarrow\left\{\xi \in E^{\perp}: \mathcal{N}(\xi) \leq\right.$ $\left.C h^{-1 / \kappa}\right\}$ which in turn shows, by Lemma 5 , that $x(t) \rightarrow$ $\pi^{-1}\left\{\xi \in E^{\perp}: \mathcal{N}(\xi) \leq C h^{-1 / \kappa}\right\}=\left\{\xi \in E^{\perp}: \mathcal{N}(\xi) \leq\right.$ $\left.C h^{-1 / \kappa}\right\}+E$ which concludes the proof.

Our aim is to apply Theorem 7 to MAS with control laws found in the literature. Let us first set some notations. The system under consideration is given by

$$
\left\{\begin{array}{c}
\dot{q}_{i}=p_{i} \\
\dot{p}_{i}=u_{i}
\end{array}\right.
$$

with $q_{i}, p_{i} \in \mathbb{R}^{n}$ and $i=1, \ldots, N$. Usually, a family of control laws $u_{i}(q, p)$ is said to achieve the consensus of the agents if in closed loop, for any $i, j, q_{i}-q_{j} \rightarrow 0$ and $p_{i}-p_{j} \rightarrow 0$. The problem with this definition is that it does not allow us to talk about stability. Let us find an equivalent formulation of the consensus. Given that, for any $i=1, \ldots, N, q_{i}$ and $p_{i} \in \mathbb{R}^{n}$, let us write the coordinates of the vector $q_{i}$ (resp. $p_{i}$ ) as $q_{i}^{k}$ (resp. $p_{i}^{k}$ ) for $k=1, \ldots, n$. For a given $k$, let us denote $r^{k}=\left(q_{1}^{k}, \ldots, q_{N}^{k}\right) \in \mathbb{R}^{N}$. Clearly, $q_{i}-q_{j} \rightarrow 0$ for all $i, j$ is equivalent to $r_{i}^{k}-r_{j}^{k} \rightarrow 0$ for all $i, j, k$. From Lemma 6 , this is equivalent to $r^{k} \rightarrow \Delta_{N}$ for all $k$. Now, let us remark that, denoting $q=\left(q_{1}^{T}, q_{2}^{T}, \ldots, q_{N}^{T}\right)^{T} \in \mathbb{R}^{n N}$, we have $q=\sum_{k=1}^{n} r^{k} \otimes e_{k}$. Hence $r^{k} \rightarrow \Delta_{N}$ for all $k$ rewrites as $q \rightarrow \operatorname{Span}\left(1_{N} \otimes e_{i}, i=1, \ldots, n\right)$. The same reasoning applied to $p=\left(p_{1}^{T}, p_{2}^{T}, \ldots, p_{N}^{T}\right)^{T} \in \mathbb{R}^{n N}$ leads to the following reformulation of the consensus in the classical sense.

Definition 8 (Attractive consensus): The system (4) with control laws $u_{i}(q, p)$ is said to achieve the attractive consensus if $\left(\begin{array}{c}q \\ p\end{array}\right) \rightarrow E$ where $g\left(\exp (\tilde{A} s) \xi_{1}, u\left(\exp (\tilde{A} s) \xi_{2}\right)\right)=\pi\left(f\left(\exp (\tilde{A} s) \xi_{1}, u\left(\exp (\tilde{A} s) \xi_{2}\right)\right)\right)$

$$
\begin{aligned}
& =\pi\left(f\left(\exp (A s) \xi_{1}, u\left(\exp (A s) \xi_{2}\right)\right)\right) \\
& =\pi\left(e^{\kappa s} \exp (A s) f\left(\xi_{1}, u\left(\xi_{2}\right)\right)\right)
\end{aligned}
$$$$
=e^{\kappa s} \exp (A s) \pi\left(f\left(\xi_{1}, u\left(\xi_{2}\right)\right)\right)
$$$$
=e^{\kappa s} \exp (\tilde{A} s) g\left(\xi_{1}, u\left(\xi_{2}\right)\right) \text {. }
$$

${ }^{1}$ See [28, Page 198] 
with $1_{N}=(1, \ldots, 1)^{T} \in \mathbb{R}^{N}$ and $\left(e_{i}\right)_{1 \leq i \leq n}$ the canonical base of $\mathbb{R}^{n}$.

Definition 9 (Stable consensus): The system (4) with control laws $u_{i}(q, p)$ is said to achieve the stable consensus if the set $E$ defined in (5) is globally asymptotically stable.

Definition 10 (Practical consensus): The system (4) with control laws $u_{i}(q, p)$ is said to achieve the practical consensus if there exists a non empty compact set $K \subset \mathbb{R}^{2 n N}$ such that $\left(\begin{array}{c}q \\ p\end{array}\right) \rightarrow K+E$.

Remark 1: Let us stress that consensus (attractive, stable or practical) does not necessarily imply the convergence of the states of the individual agents. Only the differences between the agents have to decrease and it is very common in practice for the states of the agents to diverge.

Remark 2: For continuous linear controllers which render the closed-loop MAS linear as in [29], the attractivity implies the global asymptotic stability and is enough for defining the consensus [1]. With continuous nonlinear systems, though, we know that the mere attractivity does not imply Lyapunov stability [30, Subsection 40]. In the sequel, we will only consider continuous controllers which render the closed-loop system homogeneous and we know that the attractivity implies the global asymptotic stability for homogeneous systems [26, Proposition 6.1]. In this framework of continuous homogeneous systems, Definition 8 is equivalent to Definition 9. Finally, the definition of practical consensus is an extension of the notion of practical consensus used in the literature [18].

We can now state the main result of this article dedicated to MAS.

Corollary 11: Let us consider the MAS (4). Assume known a family of continuous control laws $u_{i}(q, p)$ such that:

1) $u_{i}$ depends only on $q_{i}-q_{j}$ and $p_{i}-p_{j}$;

2) the control laws $u_{i}(q, p)$ applied to (4) achieve stable consensus;

3) there exists $1 / 2<r<1$ such that $u_{i}\left(\lambda q, \lambda^{r} p\right)=$ $\lambda^{2 r-1} u_{i}(q, p)$.

Consider an $(\eta, h)$-sampling sequence $\left(t_{k}\right)_{k \in \mathbb{N}}$. We also define $A$ the endomorphism given in the canonical base of $\mathbb{R}^{2 n N}$ by the matrix

$$
\left(\begin{array}{ll}
1 & 0 \\
0 & r
\end{array}\right) \otimes I_{n N}
$$

and $\tilde{A}$ the restriction of $A$ on $E^{\perp}$. Then, for any $\tilde{A}$ homogeneous norm $\mathcal{N}$, there exists $C>0$ such that the vector $\left(q_{1}^{T}, \ldots, q_{N}^{T}, p_{1}^{T}, \ldots, p_{N}^{T}\right)^{T} \in \mathbb{R}^{2 n N}$ converges to $\left\{\xi \in E^{\perp}\right.$ : $\left.\mathcal{N}(\xi) \leq C h^{-1 / \kappa}\right\}+E$, where $q_{i}$ and $p_{i}$ denote the solutions of

$$
\left\{\begin{array}{l}
\dot{q}_{i}(t)=p_{i}(t) \\
\dot{p}_{i}(t)=u_{i}\left(q\left(t_{k}\right), p\left(t_{k}\right)\right)
\end{array}, \quad t \in\left[t_{k}, t_{k+1}\right) .\right.
$$

Thus the the control laws $u_{i}(q, p)$ applied to (6) achieve practical consensus.

Proof. Let us apply Theorem 7. A direct computation shows that the assumption 1 of Corollary 11 implies the assumption 1 of Theorem 7, and we have obviously assumption 2 of Theorem 7 from assumption 2 of Corollary 11. The endomorphism $A$ is clearly such that $-A$ is Hurwitz. It is also straightforward to show that $E$ is a stable space for $A$ and given that $A$ is symmetric, so is $E^{\perp}$. Only the last point of assumption 3 of Theorem 7 remains to check. But indeed

$$
\begin{aligned}
\left(\begin{array}{c}
e^{r s} p \\
u\left(e^{s} \bar{q}, e^{r s} \bar{p}\right)
\end{array}\right) & =\left(\begin{array}{c}
e^{r s} p \\
e^{(2 r-1) s} u(\bar{q}, \bar{p})
\end{array}\right) \\
& =e^{(r-1) s}\left(\begin{array}{c}
e^{s} p \\
e^{r s} u(\bar{q}, \bar{p})
\end{array}\right) \\
& =e^{(r-1) s} \exp (A s)\left(\begin{array}{c}
p \\
u(\bar{q}, \bar{p})
\end{array}\right)
\end{aligned}
$$

which, setting $\kappa=r-1<0$, concludes the proof.

Remark 3: Let us recall that we assume all agents to update their control simultaneously.

No assumption is made on the graph of the agents, and the result holds for undirected as well as directed graphs, as long as the three assumptions of Corollary 11 are verified.

Assumption 3 of Theorem 7 and Corollary 11 states that the closed loop system is homogeneous of negative degree. Such systems have been notably studied in the literature for their finite-time stability properties. It is easy to see that, should we take $h=0$, that is continuous time control, we would recover these finite-time stability properties. Linear control laws would verify Assumption 3 provided that we allow $r=1$. In addition, if the control laws are designed such that, in the assumption 3, $r>1$, it is possible to show that we get local consensus instead of practical consensus (that is, exact consensus is achieved provided that the initial states of the agents are close enough). However, in practice, when the sampling period becomes large, this requires the initial states of the agents to be very close to consensus, hence drastically limiting the usefulness of this extension.

Remark 4: Let us denote $\left(\varepsilon_{i}\right)_{1 \leq i \leq N}$ the canonical base of $\mathbb{R}^{N}$ and

$$
\begin{aligned}
& M_{i j}^{q}=\left(\left(\varepsilon_{i}^{T}, 0_{N}^{T}\right)-\left(\varepsilon_{j}^{T}, 0_{N}^{T}\right)\right) \otimes I_{n}, \\
& M_{i j}^{p}=\left(\left(0_{N}^{T}, \varepsilon_{i}^{T}\right)-\left(0_{N}^{T}, \varepsilon_{j}^{T}\right)\right) \otimes I_{n} .
\end{aligned}
$$

Denoting $x=\left(q^{T}, p^{T}\right)^{T}$, a direct computation shows that $M_{i j}^{q} x=q_{i}-q_{j}$ and $M_{i j}^{p} x=p_{i}-p_{j}$. Moreover, the set $E$ defined in (5) verifies $E \subset \bigcap_{i, j} \operatorname{ker} M_{i j}^{p} \cap \operatorname{ker} M_{i j}^{q}$. Therefore, for any compact set $K \subset E^{\perp}$, we have $x(t) \rightarrow K+E \Rightarrow$ $M_{i j}^{q} x(t)=q_{i}-q_{j} \rightarrow M_{i j}^{q} K$ and then $\limsup _{t \rightarrow+\infty} \| q_{i}-$ $q_{j}\left\|\leq 2 \sup _{z \in K}\right\| z \|$. Similarly, $\lim \sup _{t \rightarrow+\infty}\left\|p_{i}-p_{j}\right\| \leq$ $2 \sup _{z \in K}\|z\|$. This ensures that, under the conclusions of Corollary 11 , the states of the agents remain asymptotically bounded by a class $\mathcal{K}$ function of $h^{-1 / \kappa}$.

Example 12: Suppose that we have an undirected and connected graph and consider the control laws for (4) given in [22]

$$
u_{i}=-\ell_{1}\left\lfloor\sum_{j=1}^{N} a_{i j}\left(q_{i}-q_{j}\right)\right]^{\alpha}-\ell_{2}\left\lfloor\sum_{j=1}^{N} a_{i j}\left(p_{i}-p_{j}\right)\right]^{\frac{2 \alpha}{(1+\alpha)}}
$$

with $0<\alpha<1, \ell_{1}>0$ and $\ell_{2}>0$. The conclusions of Corollary 11 apply to these control laws. The second assumption of Corollary 11 is the only non-trivial to show. First notice that $\sum_{j} a_{i j}\left(q_{i}-q_{j}\right)=\left(\left(\mathcal{L}_{N} \otimes I_{n}\right) q\right)_{i}$ and 
$\sum_{j} a_{i j}\left(p_{i}-p_{j}\right)=\left(\left(\mathcal{L}_{N} \otimes I_{n}\right) p\right)_{i}$, which allows us to rewrite the system under the form

$$
\left\{\begin{array}{l}
\dot{q}=p \\
\dot{p}=-\ell_{1}\left\lfloor\left(\mathcal{L}_{N} \otimes I_{n}\right) q\right\rceil^{\alpha}-\ell_{2}\left\lfloor\left(\mathcal{L}_{N} \otimes I_{n}\right) p\right\rceil^{\frac{2 \alpha}{(1+\alpha)}}
\end{array}\right.
$$

Let us now define the function

$$
V(q, p)=\ell_{1} \frac{\left\|\left(\mathcal{L}_{N} \otimes I_{n}\right) q\right\|_{1+\alpha}^{1+\alpha}}{1+\alpha}+\frac{p^{T}\left(\mathcal{L}_{N} \otimes I_{n}\right) p}{2} .
$$

A direct computation yields

$$
\begin{aligned}
\dot{V} & =\ell_{1}\left(\left(\mathcal{L}_{N} \otimes I_{n}\right) p\right)^{T}\left\lfloor\left(\mathcal{L}_{N} \otimes I_{n}\right) q\right\rceil^{\alpha}+p^{T}\left(\mathcal{L}_{N} \otimes I_{n}\right) \\
& \times\left(-\ell_{1}\left\lfloor\left(\mathcal{L}_{N} \otimes I_{n}\right) q\right\rceil^{\alpha}-\ell_{2}\left\lfloor\left(\mathcal{L}_{N} \otimes I_{n}\right) p\right\rceil^{\frac{2 \alpha}{(1+\alpha)}}\right) \\
& =-\ell_{2}\left(\left(\mathcal{L}_{N} \otimes I_{n}\right) p\right)^{T}\left\lfloor\left(\mathcal{L}_{N} \otimes I_{n}\right) p\right\rceil^{\frac{2 \alpha}{(1+\alpha)}} \\
& =-\ell_{2}\left\|\left(\mathcal{L}_{N} \otimes I_{n}\right) p\right\|_{\beta}^{\beta} \\
& \leq 0
\end{aligned}
$$

with $\beta=\frac{3 \alpha+1}{1+\alpha}$. We now use the version of the LaSalle principle given in [21, Lemma 1]: $\dot{V} \equiv 0 \Rightarrow\left(\mathcal{L}_{N} \otimes I_{n}\right) p \equiv$ $0 \Rightarrow\left(\mathcal{L}_{N} \otimes I_{n}\right) \dot{p} \equiv 0 \Rightarrow\left(\mathcal{L}_{N} \otimes I_{n}\right) q \equiv 0$. Therefore $E$ is globally asymptotically stable and Corollary 11 can be applied.

Remark 5: If the graph is supposed to be oriented, the method used in Example 12 does not work anymore. Indeed, the matrix $\mathcal{L}_{N}$ is not symmetric and the function $V$ is therefore not positive anymore.

Example 13: Suppose that we have an undirected and connected graph and consider the control laws for (4) given in [21]

$$
u_{i}=-\ell_{1} \sum_{j=1}^{N} a_{i j}\left\lfloor q_{i}-q_{j}\right\rceil^{\alpha}-\ell_{2} \sum_{j=1}^{N} a_{i j}\left\lfloor p_{i}-p_{j}\right\rceil^{\frac{2 \alpha}{(1+\alpha)}} .
$$

The conclusions of Corollary 11 apply to these control laws. This is straightforward, given that the article [21] proves the consensus for this control laws family.

\section{Simulations}

Consider the second-order MAS (6) with $N=5$ and $n=2$, i.e. 5 agents in the plane whose communications are described by the undirected graph given on Figure 1. In the following,

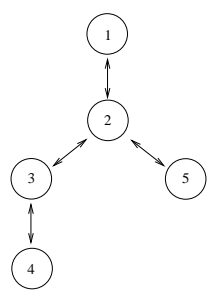

Fig. 1. Undirected graph of the 5 agents MAS

we choose constant sampling periods

$$
t_{k}=k T, \quad T>0, k \in \mathbb{N} .
$$

The initial positions $q_{i 0}$ are chosen randomly in the square $[0,100] \times[0,100]$. Consider the linear controllers

$$
u_{i}(q, p)=-\sum_{j=1}^{5} a_{i j}\left(q_{i}-q_{j}\right)-\sum_{j=1}^{5} a_{i j}\left(p_{i}-p_{j}\right)
$$

with $q_{i}=\left(q_{i_{x}}, q_{i_{y}}\right)$ and $p_{i}=\left(p_{i_{x}}, p_{i_{y}}\right)$ for $i=1, \ldots, 5$ which ensure the stable consensus (see for instance [31]). The trajectories of the sampled-data closed-loop MAS (6)-(11) with SPS (10) are drawn in Figure 2 in the plane $\left(q_{i_{x}}, q_{i_{y}}\right)$ for $T=0.1 \mathrm{~s}$. After a while, the trajectories turns into uniform linear motions. The evolutions of positions $q_{i_{x}}$ of the 5 agents are given in Figure 3 and the evolutions of velocities $p_{i_{x}}$ of the 5 agents are given in Figure 4.

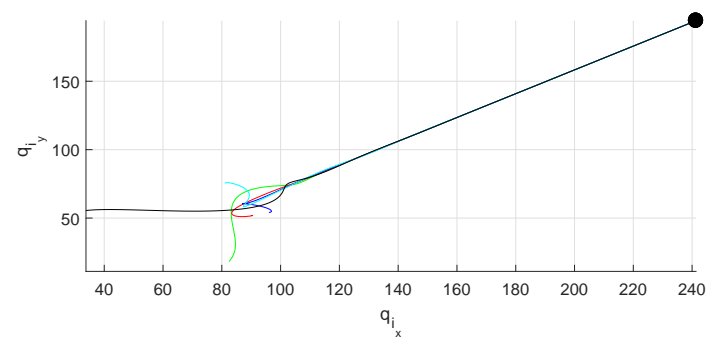

Fig. 2. Trajectories of the 5 agents in two-dimensional space $\left(q_{i_{x}}, q_{i_{y}}\right)$ with linear controllers $(11)$ and $T=0.1 \mathrm{~s}$

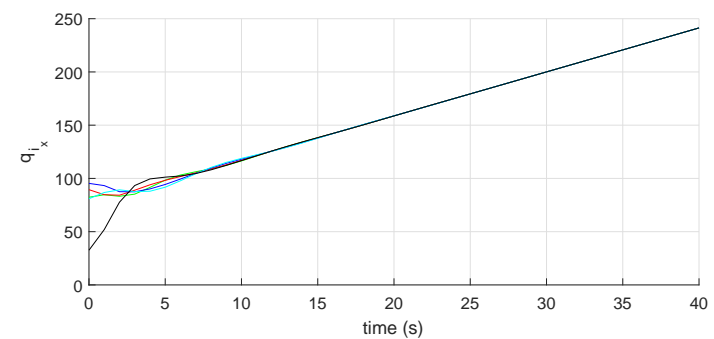

Fig. 3. Positions $q_{i_{x}}$ of the 5 agents with linear controllers (11) and $T=0.1 \mathrm{~s}$

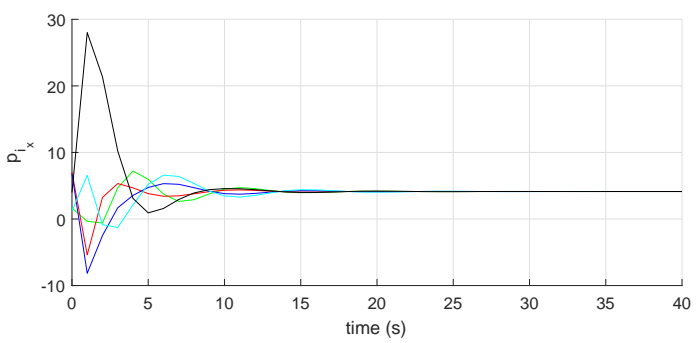

Fig. 4. Velocities $p_{i_{x}}$ of the 5 agents with linear controllers (11) and $T=0.1 \mathrm{~s}$

We know that the linear closed-loop MAS (6)-(11) with SPS (10) is asymptotically stable if and only if the matrix $\Lambda(T)$ of the linear difference equation associated with (6)-(11)-(10) and defined in [3] is Schur. For our 5 agents, $\Lambda(T)$ is Schur if and only if $T<T_{\text {Schur }}=0.4795 \mathrm{~s}$. It means that the fleet of 5 agents with the linear controllers (11)-(10) is unstable and disperses into the space when $T>0.4795 \mathrm{~s}$.

By using the controllers (7) with $\ell_{1}=\ell_{2}=1, \alpha=0.5$ and SPS (10) we obtain the practical consensus of the 5 agents as shown in Figures 5-6-7 for $T=0.5 \mathrm{~s}$. In this case, the behavior of the 5 agents reaching the practical consensus is very close to the behavior of the 5 agents reaching the stable consensus.

We obtain a result of practical consensus for higher sampling periods, as shown in Figures 8-9-10 for $T=2 \mathrm{~s}$, but the 


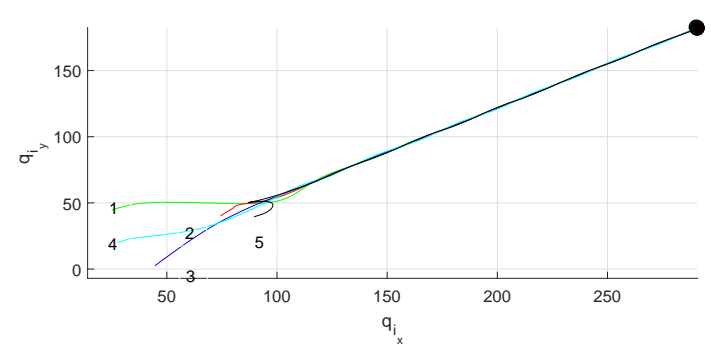

Fig. 5. Trajectories of the 5 agents in two-dimensional space $\left(q_{i_{x}}, q_{i_{y}}\right)$ with homogeneous controllers (7) and $T=0.5 \mathrm{~s}$

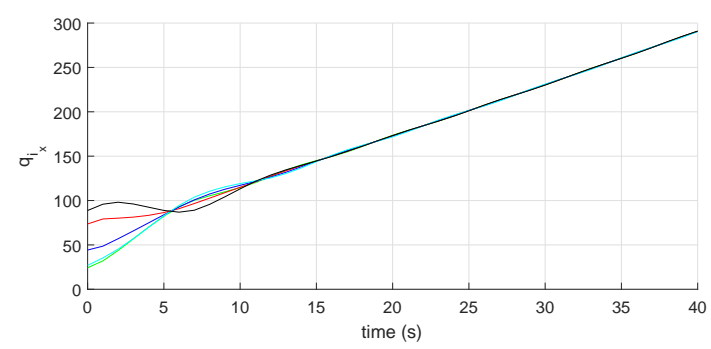

Fig. 6. Positions $q_{i_{x}}$ of the 5 agents with homogeneous controllers (7) and $T=0.5 \mathrm{~s}$

trajectories of the 5 agents are no more uniform linear motions and the velocities fluctuate. However, the fleet of 5 agents with the homogeneous controllers (7)-(10) is not unstable and does not disperse into the space.

\section{CONCLUSION}

In the article, the second-order practical consensus of MAS under synchronously variable sampling is developed by using a new nonlinear emulation strategy based on homogeneity. The results obtained with this nonlinear strategy are compared in simulation with the linear one.

\section{REFERENCES}

[1] W. Ren and R. W. Beard, Distributed consensus in multi-vehicle cooperative control, ser. Communications and Control Engineering. Springer, 2008.

[2] K.-K. Oh, M.-C. Park, and H.-S. Ahn, "A survey of multi-agent formation control," Automatica, vol. 53, pp. 424-440, 2015.

[3] L. Hetel, C. Fiter, H. Omran, A. Seuret, E. Fridman, J.-P. Richard, and S. I. Niculescu, "Recent developments on the stability of systems with aperiodic sampling: an overview," Automatica, vol. 76, pp. 309-335, 2017.

[4] Y. Cao and W. Ren, "Multi-vehicle coordination for double-integrator dynamics under fixed undirected/directed interaction in a sampled-data setting," International Journal of Robust and Nonlinear Control, vol. 20, no. 9, pp. 987-1000, 2010.

[5] W. Chen, X. Li, and L. Jiao, "Quantized consensus of second-order continuous-time multi-agent systems with a directed topology via sampled data," Automatica, vol. 49, no. 7, pp. 2236-2242, 2013.

[6] L. Cheng, Y. Wang, Z.-G. Hou, M. Tan, and Z. Cao, "Sampled-data based average consensus of second-order integral multi-agent systems: Switching topologies and communication noises," Automatica, vol. 49, no. 5, pp. 1458-1464, 2013.

[7] Y. Gao and L. Wang, "Sampled-data based consensus of continuous-time multi-agent systems with time-varying topology," IEEE Transactions on Automatic Control, vol. 56, no. 5, pp. 1226-1231, 2011

[8] N. Huang, Z. Duan, and G. R. Chen, "Some necessary and sufficient conditions for consensus of second-order multi-agent systems with sampled position data," Automatica, vol. 63, pp. 148-155, 2016

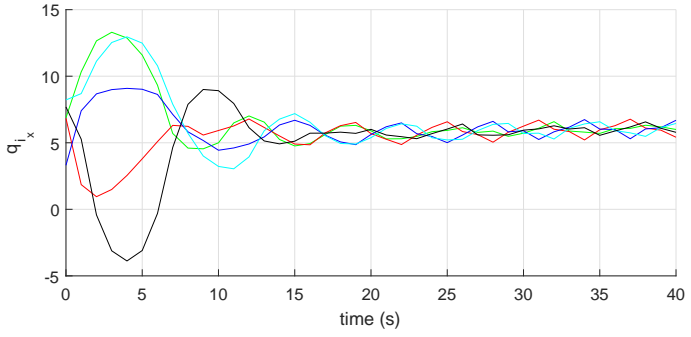

Fig. 7. Velocities $p_{i_{x}}$ of the 5 agents with homogeneous controllers (7) and $T=0.5 \mathrm{~s}$

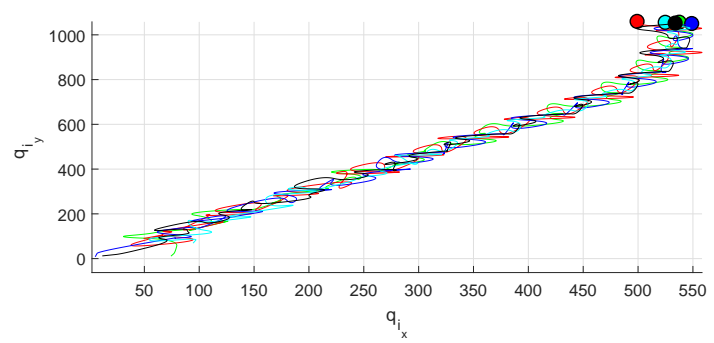

Fig. 8. Trajectories of the 5 agents in two-dimensional space $\left(q_{i_{x}}, q_{i_{y}}\right)$ with homogeneous controllers (7) and $T=2 \mathrm{~s}$

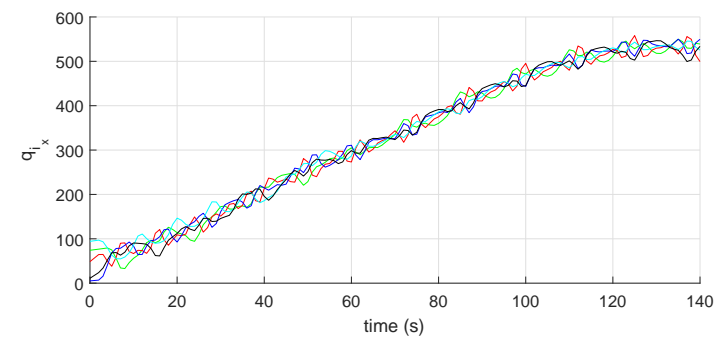

Fig. 9. Positions $q_{i_{x}}$ of the 5 agents with homogeneous controllers (7) and $T=2 \mathrm{~s}$

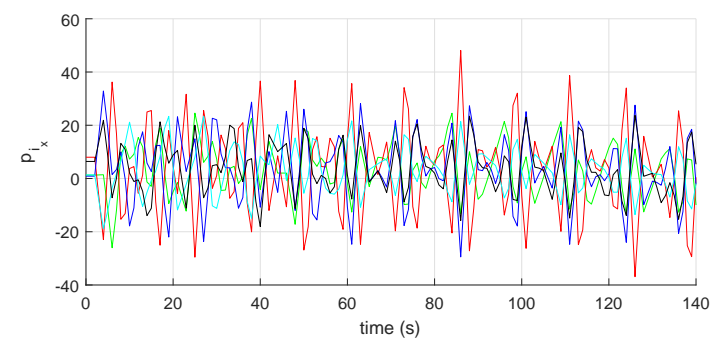

Fig. 10. Velocities $p_{i_{x}}$ of the 5 agents with homogeneous controllers (7) and $T=2 \mathrm{~s}$

[9] J. Qin and H. Gao, "A sufficient condition for convergence of sampleddata consensus for double-integrator dynamics with nonuniform and time-varying communication delays," IEEE Transactions on Automatic Control, vol. 57, no. 9, pp. 2417-2422, 2012.

[10] W. Yu, W. X. Zheng, G. Chen, W. Ren, and J. Cao, "Second-order consensus in multi-agent dynamical systems with sampled position data," Automatica, vol. 47, no. 7, pp. 1496-1503, 2011.

[11] G. Wen, Z. Duan, W. Yu, and G. Chen, "Consensus of multi-agent systems with nonlinear dynamics and sampled-data information: a delayed-input approach," International Journal of Robust and Nonlinear Control, vol. 23, no. 6, pp. 602-619, 2013.

[12] J. Qin, C. Yu, and S. Hirche, "Stationary consensus of asynchronous discrete-time second-order multi-agent systems under switching topol- 
ogy," IEEE Transactions on Industrial Informatics, vol. 8, no. 4, pp. 986-994, 2012.

[13] X. Ge, F. Yang, and Q.-L. Han, "Distributed networked control systems: A brief overview," Information Sciences, vol. 380, pp. 117-131, 2017.

[14] X. Ge, Q.-L. Han, D. Ding, X.-M. Zhang, and B. Ning, "A survey on recent advances in distributed sampled-data cooperative control of multi-agent systems," Neurocomputing, vol. 275, pp. 1684-1701, 2018.

[15] E. Bernuau, E. Moulay, and P. Coirault, "Stability of homogeneous nonlinear systems with sampled-data inputs," Automatica, vol. 85, pp. 349-355, 2017.

[16] A. Bemporad, M. Heemels, and M. Johansson, Networked control systems, ser. Lecture Notes in Control and Information Sciences. Springer, 2010, vol. 406.

[17] D. Nešić, A. R. Teel, and D. Carnevale, "Explicit computation of the sampling period in emulation of controllers for nonlinear sampled-data systems," IEEE Transactions on Automatic Control, vol. 54, no. 3, pp. 619-624, 2009

[18] X. Dong, J. Xi, Z. Shi, and Y. Zhong, "Practical consensus for highorder linear time-invariant swarm systems with interaction uncertainties, time-varying delays and external disturbances," International Journal of Systems Science, vol. 44, no. 10, pp. 1843-1856, 2013.

[19] S. P. Bhat and D. S. Bernstein, "Finite-time stability of continuous autonomous systems," SIAM Journal on Control and Optimization, vol. 38 , no. 3 , pp. 751-766, 2000 .

[20] S. Li, H. Du, and X. Lin, "Finite-time consensus algorithm for multiagent systems with double-integrator dynamics," Automatica, vol. 47, no. 8, pp. 1706-1712, 2011.

[21] X. Wang and Y. Hong, "Finite-time consensus for multi-agent networks with second-order agent dynamics," in 17th IFAC World Congress. Seoul, Korea: Elsevier, 2008, pp. 15 185-15 190.

[22] Y. Zhao, Z. Duan, G. Wen, and Y. Zhang, "Distributed finite-time tracking control for multi-agent systems: an observer-based approach," Systems \& Control Letters, vol. 62, no. 1, pp. 22-28, 2013.

[23] F. Xiao and T. Chen, "Sampled-data consensus for multiple double integrators with arbitrary sampling," IEEE Transactions on Automatic Control, vol. 57, no. 12, pp. 3230-3235, 2012.

[24] N. P. Bhatia and G. P. Szegö, Stability theory of dynamical systems, ser. Classics in Mathematics. Springer, 2002, vol. 161.

[25] L. P. Rothschild and E. M. Stein, "Hypoelliptic differential operators and nilpotent groups," Acta Mathematica, vol. 137, no. 1, pp. 247-320, 1976.

[26] S. P. Bhat and D. S. Bernstein, "Geometric homogeneity with applications to finite-time stability," Mathematics of Control, Signals and Systems, vol. 17, no. 2, pp. 101-127, 2005.

[27] M. Kawski, "Geometric homogeneity and stabilization," in Nonlinear Control Systems Design. Elsevier, 1995, pp. 147-152.

[28] M. C. Turner and D. G. Bates, Mathematical Methods for Robust and Nonlinear Control, ser. Lecture Notes in Control and Information Sciences. Springer, 2007, vol. 367.

[29] W. Ren and E. Atkins, "Distributed multi-vehicle coordinated control via local information exchange," International Journal of Robust and Nonlinear Control, vol. 17, no. 10-11, pp. 1002-1033, 2007.

[30] W. Hahn and A. P. Baartz, Stability of motion, ser. Die Grundlehren der mathematischen Wissenschaften. Springer, 1967, vol. 138.

[31] W. Yu, L. Zhou, X. Yu, J. Lü, and R. Lu, "Consensus in multiagent systems with second-order dynamics and sampled data," IEEE Transactions on Industrial Informatics, vol. 9, no. 4, pp. 2137-2146, 2013. 